\title{
Comparación de la densidad mineral ósea en futbolistas y árbitros de nivel profesional
}

\section{Comparison of bone mineral density in soccer players and professional referees}

\author{
Ricardo López García*, José Omar Lagunes Carrasco, Luis Enrique Carranza García y Ricardo Navarro Orocio
}

Universidad Autónoma de Nuevo León (México).

\begin{abstract}
Resumen: El objetivo de este estudio fue comparar la densidad mineral ósea (DMO) de los futbolistas y árbitros de nivel profesional. Se realizaron mediciones de la densidad mineral del hueso con el equipo de absorciometría dual de rayos X (DEXA) en el cual se evaluaron las regiones de la columna, cadera, costillas, brazos, piernas y cuerpo completo a 27 futbolistas (19.81 \pm 1.14 años de edad) y 22 árbitros $(24.95 \pm 3.54$ años de edad $)$ de la segunda división de la liga profesional de fútbol MX de México. Se utilizo el programa estadístico SPSS (21.0), utilizando la prueba para muestras independientes de la $t$ de Student, considerando el valor de significancia de $p \leq .05$. Los futbolistas lograron una mayor DMO que los árbitros en la mayoría de las regiones del cuerpo, encontrándose solamente diferencia significativa en las piernas y en la cadera $(p \leq .05)$. La alta mineralización de las piernas de los futbolistas se debe al alto contacto de repetidas ocasiones con el balón de juego, aunque también se obtuvo un alta DMO en los árbitros que en otros deportes de otros estudios. Concluyendo así que la práctica del arbitraje en el fútbol es positivo para el fortalecimiento del hueso. Sin embargo hacen falta realizar más estudios sobre la DMO en árbitros, réferi y jueces en el fútbol y en otras disciplinas.

Palabras clave: Futbolistas, árbitros, densidad mineral ósea, DEXA.
\end{abstract}

Abstract: The aim of this study was to compare bone mineral density (BMD) of soccer players and professional referees. There were made bone mineral density measurements with the dual x-ray absorptiometry equipment (DEXA) to 27 players $(19.81 \pm 1.14$ years old) and 22 referees $(24.95 \pm$ 3.54 years old) from the second division of the Mexican professional soccer league (Liga MX) where the areas of the spine, hip, ribs, arms, legs and full body were evaluated. It was used the statistical program SPSS (21.0), using the test for independent samples of the $t$ of Student, considering the significance value of $\mathrm{p} \leq .05$. The players achieved a higher BMD than the referees in most regions of the body, finding only significant difference in the legs and hip $(\mathrm{p} \leq .05)$. The high mineralization of the legs of the players is due to the high contact repeatedly to the game ball, although a higher BMD was also obtained in the referees than in other sports of other studies. Thus concluding that the practice of refereeing in football is positive for bone strengthening. However, more studies on BMD in referees, officials and judges in football and in other disciplines are needed.

Keywords: soccer players, referees, bone mineral density, DEXA.

\section{Introducción}

Es evidente que la práctica de la actividad física favorece la densidad mineral en el hueso (Christoffersen et al., 2015; Cervinka, Tittweger, Hyttinen, Felsenberg, \& Sievänen, 2011), sin dejar de lado de que la mineralización y la desmineralización puede también obtenerse por la predisposición genética, el control hormonal, la conducta del sistema endocrino y el comportamiento de la alimentación en la dieta (Manuel Gómez, 2006; Oh et al., 2005; Sun, Davies, Blair, Abe, \& Zaidi, 2006). La práctica de algunas disciplinas deportivas favorece el aumento del mineral en el sistema óseo. Principalmente disciplinas en el que el hueso tiene una gran carga muscular como levantamiento de pesas (Lafforgue, 2013), disciplinas en el cual hay un impacto con el hueso como los deportes de combates, tenis, gimnasia y voleibol, y disciplinas con ejercicios aeróbicos como correr (Abrahi et al., 2016; Kontulainen et al., 2001). Mientras deportes en el cual no existe un contacto con el hueso como la natación y el ciclismo suelen tener un efecto negativo de la mineralización ósea (Sherk et

Dirección para correspondencia [Correspondence address]: Ricardo López García. Universidad Autónoma de Nuevo León (México).

E-mail: ricardo78-82@hotmail.com al., 2014; Cadore, Brentano, \& Kruel, 2005; Silva, Goldberg, Teixeira, \& Dalmas, 2011; Abrahin et al., 2016).

En la prctica del fútbol se realizan distintos movimientos como saltos, arranques, caídas y cambio de direcciones, en el cual fortalecen la mineralización del hueso en especial los miembros inferiores (Calbet, Dorado, Díaz-Herrera, \& Rodríguez-Rodríguez, 2001; Scott, Khan, Duronio, \& Hart, 2008). Además, es un deporte en el cual se practica con una pelota en el cual tiene contacto con las piernas, dando una fuerza de reacción en el esqueleto y así considerándolo como un deporte de alto impacto (Vicente-Rodríguez et al., 2004; Abrahin et al., 2016). Para poder llevar a cabo un partido de fútbol en el campo de juego es fundamental contar con árbitros para poder ejercer la autoridad durante la competición en juego, en el cual son exigidos con carreras de alta velocidad, arranques y cambios de direcciones durante todo el partido, para así poder valorar y calificar en tiempo todas las jugadas. El estado físico de un árbitro en un partido es de alta demanda, ya que a nivel profesional alcanzan a recorrer grandes distancia durante un partido, en el cual depende mucha la posición, por ejemplo un árbitro central puede alcanzar hasta $14 \mathrm{~km}$ en un juego mientras un árbitro asistente hasta 
$6.5 \mathrm{~km}$ (Weston, \& Brewer, 2002; Weston et al., 2012; Mallo, Navarro, Aranda, \& Helsen, 2009).

Está claro que en un partido de fútbol tanto el jugador como el árbitro suelen tener idénticos movimientos mecánicos así como la misma exigencia física. Solo mostrando la diferencia en que los futbolistas suelen tener un contacto de impacto en los miembros inferiores que es con el balón de juego. Concluyendo así que el futbolista suele practicar un deporte de alto impacto, y el árbitro de fútbol suele considerarse como una práctica en que no hay un impacto considerable con la estructura ósea (Vicente-Rodriguez et al., 2004). Se han encontrado muchos estudios sobre la valoración corporal en futbolista y árbitros de fútbol, algunos cuantos sobre la evaluación de la DMO en futbolistas, pero no se han encontrado estudios en el cual se valore la densidad mineral del hueso en los árbitros. Por eso nuestro objetivo es comparar la DMO de los futbolistas y árbitros de nivel profesional de la liga de fútbol en México.

\section{Material y método}

\subsection{Participantes}

Este estudio fue de tipo comparativo y transversal, con una muestra de 27 futbolistas $(19.81 \pm 1.14$ años de edad) y 22 árbitros $(24.95 \pm 3.54$ ańos de edad) de la segunda división de la liga profesional de fútbol MX de México. Ambos grupos se les entrego una carta de consentimiento informado, en la cual los sujetos firmaron para así poder participar.

\subsection{Instrumentos}

Se realizaron mediciones antropométricas de peso corporal y estatura, siguiendo las normas del manual de protocolo de la Sociedad Internacional de Avances de la Cineantropometría (ISAK) (Marfell-Jones, Stewart, \& De Ridder, 2012). Para el peso corporal se utilizó la báscula Tanita TBF-410 (0 - 200 $\mathrm{kg} \pm 0.01 \mathrm{~kg}$ ) y para la estatura se utilizó el estadiómetro Seca $213(20-205 \mathrm{~cm} \pm 5 \mathrm{~mm})$. En la valoración de la DMO se usó el equipo de la DEXA de la marca General Electric ([GE Healthcare Lunar Technology bone radiodensitometry] con software enCORE Modelo LU43616ES).

\subsection{Procedimiento}

Se convocó a los deportistas con un ayuno de no más de 8 horas y sin haber realizado ninguna actividad física. Poste- riormente, personas certificadas por el ISAK realizaron las mediciones del peso corporal y la estatura. Después de haber hecho las mediciones antropométricas a los deportistas, pasaron al laboratorio para medirles la DMO, citándolos con la ropa imprescindible y sin aportar accesorios de metal para a continuación colocarlos en posición supina sobre la mesa de la exploración de la DEXA. La estatura y el peso corporal fueron obtenidos previamente por la báscula Tanita. Se introdujo en el software del DEXA la edad, para dar comienzo a la medición del escáner, a través del cual se valoraron las regiones de la columna, cadera, costillas, brazos, piernas y cuerpo completo. En los brazos y piernas se midieron las extremidades del lado derecho e izquierdo logrando el valor medio de ambas. Una vez terminada la medición con el escáner, el software de la DEXA nos arrojo los gramos de mineral de cada región en centímetros cuadrados $\left(\mathrm{g} / \mathrm{cm}^{2}\right)$, así como los valores de referencia de la DMO establecidas por la Organización Mundial de la Salud (OMS), en la cual una T Score mayor o igual a -1 lo clasifica como "adecuado", entre -1 y -2.5 lo clasifica como "osteopenia” y un menos o igual de -2.5 lo clasifica como "osteoporosis".

\subsection{Análisis estadístico}

El análisis estadístico se realizó con el programa del paquete SPSS (IBM, SPSS Statistics Versión 21.0). La estadística descriptiva se presentó como las medias y desviación estándar de las variables de medición. En la distribución normal de las variables de estudio se utilizó la prueba de KolmogorovSmirnov. Para comparar la DMO de las regiones (columna, cadera, costillas, brazos, piernas y cuerpo completo) entre los futbolistas y árbitros se determinó con la prueba para muestras independientes $t$ de Student, considerando el valor de significancia de $p \leq .05$.

\section{Resultados}

En la tabla 1 se muestran los resultados de las mediciones básicas, en los cuales los árbitros presentan un mayor peso corporal y un mayor IMC que los futbolistas, localizándose dentro del rango de normalidad del IMC (18.5 a 24.9). En la masa grasa los árbitros obtuvieron mayor porcentaje y kilogramos de este componente que los futbolistas, encontrándose diferencias significativas $(p \leq .05)$ 
Tabla 1. Mediciones básicas de los futbolistas y árbitros.

\begin{tabular}{lccc}
\hline Mediciones básicas & Futbolistas & Árbitros & Valor de $p$ \\
\hline Edad & $19.81 \pm 1.14$ & $24.95 \pm 3.54$ & $73.49 \pm 7.87$ \\
Peso $(\mathrm{kg})$ & $71.70 \pm 7.21$ & $174.56 \pm 5.98$ & .410 \\
Estatura $(\mathrm{cm})$ & $175.65 \pm 6.65$ & $24.17 \pm 2.83$ & .553 \\
IMC $\left(\mathrm{kg} / \mathrm{m}^{2}\right)$ & $23.23 \pm 2.02$ & $21.25 \pm 3.71$ & .185 \\
Grasa $(\%)$ & $15.47 \pm 3.50$ & $15.18 \pm 3.84$ & .000 \\
Grasa $(\mathrm{kg})$ & $10.91 \pm 3.53$ & & .000 \\
\hline
\end{tabular}

En los resultados de la densidad mineral del hueso, se puede observar (tabla 2) que los futbolistas obtuvieron mayor DMO que los árbitros en las regiones de las costillas, tronco, cuerpo completo, cadera y piernas, encontrándose diferencias significativas solo en las regiones de la cadera y piernas $(p \leq$
.05). Los árbitros mostraron mayor DMO en las regiones de la columna y los brazos, pero no se encontraron diferencias significativas. En los niveles de la DMO, los futbolistas y los árbitros se encuentran en el nivel adecuado con respecto a los valores de normalidad de la T Score (mayor o igual a -1).

Tabla 2. Mediciones de la DMO de las regiones del cuerpo de los futbolistas y árbitros.

\begin{tabular}{lccc}
\hline Regiones del cuerpo & Futbolistas & Árbitro & Valor de $p$ \\
\hline Columna $\left(\mathrm{g} / \mathrm{cm}^{2}\right)$ & $1.208 \pm 0.107$ & $1.225 \pm 0.109$ & .576 \\
Cadera $\left(\mathrm{g} / \mathrm{cm}^{2}\right)$ & $1.414 \pm 0.111$ & $1.339 \pm 0.106$ & .020 \\
Costillas $\left(\mathrm{g} / \mathrm{cm}^{2}\right)$ & $0.924 \pm 0.103$ & $0.913 \pm 0.091$ & .722 \\
Tronco $\left(\mathrm{g} / \mathrm{cm}^{2}\right)$ & $1.185 \pm 0.083$ & $1.153 \pm 0.085$ & .201 \\
Brazos $\left(\mathrm{g} / \mathrm{cm}^{2}\right)$ & $0.948 \pm 0.123$ & $0.996 \pm 0.250$ & .384 \\
Piernas $\left(\mathrm{g} / \mathrm{cm}^{2}\right)$ & $1.558 \pm 0.096$ & $1.468 \pm 0.083$ & .001 \\
Cuerpo completo $\left(\mathrm{g} / \mathrm{cm}^{2}\right)$ & $1.381 \pm 0.092$ & $1.368 \pm 0.087$ & .621 \\
\hline
\end{tabular}

\section{Discusión}

Medir la densidad mineral del hueso es también conocer el estado morfológico del sujeto, por eso es importante realizar estas evaluaciones a deportistas para poder conocer el estado físico, que nos ayudara a monitorear el rendimiento, la salud y así como prevención de fracturas. El fútbol es considerado como un deporte de alto impacto (Sherk et al., 2014), pero la práctica del arbitraje en el fútbol es considerado solamente como aplicar el reglamento en la cancha, sin conocer que arbitrar un partido de fútbol demanda una gran exigencia física. Por lo que el objetivo en este estudio fue comparar la DMO de los futbolistas y árbitros profesionales de nivel profesional.

Las evaluaciones de la DMO de las diferentes regiones del cuerpo entre los futbolistas y árbitros nos das a conocer las diferentes técnicas de carga mecánicas que exige cada disciplina, alterando la formación ósea del deportista. Normalmente estas dos actividades físicas (fútbol y arbitraje) requieren más la participación de las extremidades inferiores, y más los futbolistas por el alto impacto de las piernas contra el balón, prediciendo que estas regiones del cuerpo tendrían más DMO los futbolistas que los árbitros debido al este impacto y a la realización de algunos movimiento mecánicos como el saltar, confirmando así nuestra hipótesis en este estudio, en la que la DMO de los miembros inferiores de los futbolistas fue significativamente mayor $(\mathrm{p}=.001)$ que los árbitros. Al igual si lo comparamos con otros deportes, los estudios dicen que
DMO de las piernas en futbolistas es mayor que en deportes como el tenis, triatlón, ciclismo y natación (McClanahan, 2012; Morel, Combe, Francisco, \& Bernard, 2001; Silva et al., 2011; Calbet et al., 2001; Wittich et al., 1998). Esto no significa que la práctica del arbitraje no sea beneficioso en el fortalecer la densidad en las piernas, dado que los resultados de la DMO de nuestros árbitros son superiores que en otros deportes como atletismo, ciclismo y natación (McCLANAHAN, 2012; Morel et al., 2001; Silva et al., 2011; Nichols, \& Rauh, 2011; Fredericson, 2007; Czeczelewski, Długołęcka, Czeczelewska, \& Raczyńska, 2013).

Algunos trabajos (McClanahan, 2012; Morel et al., 2001) evaluaron la DMO de los miembros superiores e inferiores a diferentes grupos de deportes, en los cuales los resultados de la región inferior de los deportes de beisbol, golf y tenis se encuentran por debajo de los valores de nuestros futbolistas y árbitros. Esto debido a la poca demanda de impacto que requiere los miembros inferiores. Además estos deportes ya mencionados implican una carga asimétrica en la región inferior, en la cual esfuerzan un miembro más que la otra, que trae como consecuencias valores no muy alto en la DMO.

Los deportes como el fútbol americano y el rugby suelen tener un alto contacto durante la práctica, expresando así que su DMO es muy elevada tanto en las piernas como en los brazos, tal como lo demuestran los estudios de $\mathrm{McCla}-$ nahan (2012) y de Morel et al. (2001) en los cuales la DMO de los brazos es elevada por la alta participación de esta re- 
gión. Todo lo contrario ocurre en deportes donde también suelen tener una alta participación en la región de los brazos como el beisbol, tenis, golf y el basquetbol, y esto puede deberse por que involucran la carga a un solo lado del cuerpo por ser una actividad asimétrica, dejando poca participación el otro lado de la región del brazo. En el fútbol y el arbitraje no suelen usar mucho esta zona durante la práctica, justa razón por la cual se obtuvo una DMO baja en los brazos de nuestros futbolistas $\left(0.947 \mathrm{~g} / \mathrm{cm}^{2}\right)$ y árbitros $(0.996 \mathrm{~g} /$ $\mathrm{cm}^{2}$ ) en comparación de los jugadores de fútbol americano $\left(1.055 \mathrm{~g} / \mathrm{cm}^{2}\right)$ y rugby $\left(1.070 \mathrm{~g} / \mathrm{cm}^{2}\right)$ (McClanahan, 2012; Morel et al.,2001).

Otro deporte en la cual se suele utilizar mucho los brazos durante la práctica es la natación, aunque se han encontrado estudios como el de Maimoun et al. (2004) y Morel et al. (2001) en los cuales los nadadores obtuvieron valores bajos de la $\mathrm{DMO}$ en esta región en comparación de otros deportes como el triatlón y ciclismo, y hasta el de nuestros futbolistas y árbitros, que son deportes en la cual su participación de la región superior es poca activa, no hay que olvidar que la natación es considerado como un deporte de bajo impacto sobre el esqueleto (Silva et al., 2011), ya que sus movimiento mecánicos en el agua suelen hacerse en una baja gravedad, justificando que la práctica de este deporte puede ser algo ne- gativo para el fortalecimiento del hueso en todos las regiones del cuerpo.

\section{Conclusiones}

En conclusión, los futbolistas arrojaron una mayor DMO en los miembros inferiores que los árbitros, esto puede deberse a que la práctica del fútbol genera una carga mecánica sobre el hueso de las piernas, además de tener un alto contacto en repetidas ocasiones con el balón de juego, es beneficioso para el fortalecimiento de este punto anatómico. Aunque los niveles de la DMO de las piernas de nuestros árbitros están por encima de otros deportes de algunos estudios. Esto queda en manifestó que la práctica del arbitraje en el fútbol es positivo para el fortalecimiento del hueso, no obstante no podemos descartar que otras causas como la alimentación, estilo de vida y cuestiones genéticas, pudieron haber favorecido los resultados obtenidos. Queda mucho por trabajar en esta grupo que son los árbitros, por lo que investigaciones futuras se propone realizar evaluaciones longitudinales de la DMO junto con al monitoreo de las capacidades físicas y alimentación, que puedan permitir tener un nivel de conocimiento más claro acerca de la mineralización del hueso en este tipo de actividades.

\section{Referencias}

1. Abrahin, O., Rodrigues, R. P., Marçal, A. C., Alves, E. A. C., Figueiredo, R. C., \& Sousa, E. C. D. (2016). Swimming and cycling do not cause positive effects on bone mineral density: a systematic review. Revista brasileira de reumatologia, 56(4), 345-351.

2. Cadore, E. L., Brentano, M. A., \& Kruel, L. F. M. (2005). Effects of the physical activity on the bone mineral density and bone remodelation. Revista Brasileira de Medicina do Esporte, 11(6), 373-379.

3. Calbet, J. A., Dorado, C., Diaz-Herrera, P., \& Rodriguez-Rodriguez, L. P. (2001). High femoral bone mineral content and density in male football (soccer) players. Medicine and science in sports and exercise, 33(10), 1682-1687.

4. Cervinka, T., Rittweger, J., Hyttinen, J., Felsenberg, D., \& Sievänen, H. (2011). Anatomical sector analysis of load-bearing tibial bone structure during 90-day bed rest and 1-year recovery. Clinical physiology and functional imaging, 31(4), 249-257.

5. Christoffersen, T., Winther, A., Nilsen, O. A., Ahmed, L. A., Furberg, A. S., Grimnes, G., ... \& Emaus, N. (2015). Does the frequency and intensity of physical activity in adolescence have an impact on bone? The Tromsø Study, Fit Futures. BMC sports science, medicine and rehabilitation, 7(1), 26.

6. Czeczelewski, J., Długołęcka, B., Czeczelewska, E., \& Raczyńska, B. (2013). Intakes of selected nutrients, bone mineralisation and density of adolescent female swimmers over a three-year period. Biology of sport, 30(1), 17.

7. Fredericson, M., Chew, K., Ngo, J., Cleek, T., Kiratli, J., \& Cobb, K. (2007). Regional bone mineral density in male athletes: a comparison of soccer players, runners and controls. British journal of sports medicine, 41(10), 664-668.

8. Kontulainen, S., Kannus, P., Haapasalo, H., Sievänen, H., Pasanen, M., Heinonen, A., ... \& Vuori, I. (2001). Good maintenance of exercise-in- duced bone gain with decreased training of female tennis and squash players: a prospective 5-year follow-up study of young and old starters and controls. Journal of Bone and Mineral Research, 16(2), 195-201.

9. Lafforgue, P. (2013). Fracturas por sobrecarga (fracturas por fatiga y fracturas por insuficiencia ósea). EMC-Aparato Locomotor, 46(3), 1-12.

10. Maimoun, L., Mariano-Goulart, D., Couret, I., Manetta, J., Peruchon, E., Micallef, J. P., ... \& Leroux, J. L. (2004). Effects of physical activities that induce moderate external loading on bone metabolism in male athletes. Journal of sports sciences, 22(9), 875-883.

11. Mallo, J., Navarro, E., Aranda, J. M. G., \& Helsen, W. F. (2009). Activity profile of top-class association football referees in relation to fitnesstest performance and match standard. Journal of sports sciences, 27(1), $9-17$.

12. Manuel Gomez, J. (2006). The role of insulin-like growth factor I components in the regulation of vitamin D. Current Pharmaceutical Biotechnology, 7(2), 125-132.

13. Marfell-Jones, M. J., Stewart, A. D., \& De Ridder, J. H. (2012). International standards for anthropometric assessment.

14. McClanahan, B. S., Harmon-Clayton, K., Ward, K. D., Klesges, R. C., Vukadinovich, C. M., \& Cantler, E. D. (2002). Side-to-side comparisons of bone mineral density in upper and lower limbs of collegiate athletes. Journal of strength and conditioning research, 16(4), 586.

15. Morel, J., Combe, B., Francisco, J., \& Bernard, J. (2001). Bone mineral density of 704 amateur sportsmen involved in different physical activities. Osteoporosis International, 12(2), 152-157.

16. Nichols, J. F., \& Rauh, M. J. (2011). Longitudinal changes in bone mineral density in male master cyclists and nonathletes. The Journal of Strength \& Conditioning Research, 25(3), 727-734.

17. Oh, K. W., Lee, W. Y., Rhee, E. J., Baek, K. H., Yoon, K. H., Kang, M. I., ... \& Yoo, H. J. (2005). The relationship between serum resistin, 
leptin, adiponectin, ghrelin levels and bone mineral density in middleaged men. Clinical endocrinology, 63(2), 131-138.

18. Sherk, V. D., Barry, D. W., Villalon, K. L., Hansen, K. C., Wolfe, P., \& Kohrt, W. M. (2014). Bone loss over one year of training and competition in female cyclists. Clinical journal of sport medicine: official journal of the Canadian Academy of Sport Medicine, 24(4), 331.

19. Silva, C. C., Goldberg, T. B., Teixeira, A. S., \& Dalmas, J. C. (2011). The impact of different types of physical activity on total and regional bone mineral density in young Brazilian athletes. Journal of sports sciences, 29(3), 227-234.

20. Scott, A., Khan, K. M., Duronio, V., \& Hart, D. A. (2008). Mechanotransduction in human bone. Sports Medicine, 38(2), 139-160.

21. Sun, L. I., Davies, T. F., Blair, H. C., Abe, E., \& Zaidi, M. (2006). TSH and bone loss. Annals of the New York Academy of Sciences, 1068(1), 309318.
22. Vicente-Rodriguez, G., Ara, I., Perez-Gómez, J., Serrano-Sanchez, J.A., Dorado, C., \& Calbet, J.A. (2004). High femoral bone mineral density accretion in prepubertal soccer players. Medicine and science in sports and exercise, 36(10), 1789-1795.

23. Weston, M., \& Brewer, J. (2002). A study of the physiological demands of soccer refereeing. Journal of Sports Sciences.

24. Weston, M., Castagna, C., Impellizzeri, F. M., Bizzini, M., Williams, A. M., \& Gregson, W. (2012). Science and medicine applied to soccer refereeing. Sports medicine, 42(7), 615-631.

25. Wittich, A., Mautalen, C. A., Oliveri, M. B., Bagur, A., Somoza, F., \& Rotemberg, E. (1998). Professional football (soccer) players have a markedly greater skeletal mineral content, density and size than ageand BMI-matched controls. Calcified tissue international, 63(2), 112117. 